\title{
Form-finding of pierced vaults and digital fabrication of scaled prototype ${ }^{\star *}$
}

https://doi.org/10.1515/cls-2021-0020

Received Sep 30, 2020; accepted Dec 19, 2020

\begin{abstract}
The new serious consideration to masonry and non-metallic structures evidenced their direct prospective to be, even in the present days, advanced architectural and engineering solutions. In the present paper, a form finding for a cement based tessellated pierced vault is studied. The multi-body rope approach (MRA) was used to define compression-only vault optimal shapes. Successively, the thrust network analysis (TNA) was implemented by Rhinovault for a further validation of the shape and the definition of different tessellation meshes of the surfaces, according to different hole pattern configuration. Different piercing percentage of the vaults were considered and compared for the best solution identification. In addition, the geometrical solutions were analyzed by means of global stability analysis, taking into account the different positions of the holes. Furthermore, 3D printing with a Fuse Deposition Modeling (FDM) technique in polylactide (PLA) material (completely eco-friendly) is used for the construction of the formworks of the cement based blocks (dowels) useful for the assembly of a vault scaled prototype. The prototype of the vault, characterized by a certain piercing percentage was subjected to different loading conditions and monitored by a non-contact device based on the Digital Image Correlation (DIC) technique. The 3D-DIC was performed to recognize the structural behavior during the loading process of the model (prototype). DIC measurements were used to recognize in advance the critical condition of the vault under loading and the displacement measurements were correlated to the different loading phases up to the collapse condition.
\end{abstract}

Keywords: Masonry vault, additive manufacturing, stability evaluation, buckling analysis, digital image correlation

\footnotetext{
`Corresponding Author: Amedeo Manuello Bertetto: Department of Structural, Geotechnical and Building Engineering, Politecnico di Torino, Torino, Italy; Email: amedeo.manuellobertetto@polito.it

Federico Riberi: Maffeis Engineering SpA, Via Mignano 26 - Solagna (Vi), Italy
}

כ Open Access. (c) 2021 A. M. Bertetto and F. Riberi, published by De Gruyter. Attribution 4.0 License

\section{Introduction}

The new attention to masonry structures evidenced their continuous potential to be an actual architectural and engineering solution revisited and rethought by innovative realizations and design tools [1-5]. The increasing interest to study ancient masonry vaults, erected all over the world, gave back today a renewed necessity to suggest this kind of structures to cover innovative and sustainable buildings. At the same time, the stability condition of ancient and new masonry vaults represents a fascinating and crucial area of research in several countries such as Italy. Over times, the increasing importance of computer based design and modelling procedures changed the link between architecture and structural concepts generating holistic approaches for shape, material and structures [1-3]. Through new formfinding procedures based on automatic tools and models also the digital fabrication methods played a fundamental role in the realization of non-conventional and free form architectures [1-5]. In order to realize an efficient design for these unconventional structures the sharing of computational tools and techniques, gained a great importance among researchers and components of the design community $[1,4]$. To allow an in-built strategy to design problems with solid engineering constraints through visual representation $[1,5]$ and real-time reaction $[1,6]$ of structural information became a crucial aspect.

In the last decades many papers discussed the problem of doubly-curved shells [6] and curved surface design within the rigorous framework of funicular, compressiononly vault design $[1,7-9]$. Historically, hanging models and graphic statics were used to design domed structures. At the beginning of the $20^{\text {th }}$ century, Antoni Gaudi used hanging scaled reproductions in the design process of the Crypt of Colònia Güell [1, 6], Frei Otto and his team used hanged models to find the form for the lattice shell in Mannheim [8], and Swiss engineer Heinz Isler designed his concrete shells using hanged cloth mockups $[1,10]$. More recently, shape

$\star \star$ Paper included in the Special Issue entitled: Shell and Spatial Structures: Between New Developments and Historical Aspects This work is licensed under the Creative Commons 
optimization of grid shells has been carried out using different techniques $[11,12,14]$. At the same time, discrete truss topology method [15], graph based design [16], simulated annealing [17], and cut-and-branch methods [18] have been used. Moreover, genetic algorithms have been recently employed for the optimization of three-dimensional discrete system, such as spatial structures, planar structures and geodetic domes [19]. Multi-objectives optimization schemes have been developed by Winslow for free form grid shell constituted by elements with variable orientation [20]. Form-finding approaches, such as the force density method [18] and the dynamic relaxation (DR) [19], have been applied to weightless configurations. Among these last kinds of systems, Kilian and Ochsendorf [11] proposed a shape-finding tool for statically determined systems based on particle-spring model. Block and Ochsendorf proposed the thrust network analysis to establish the shape of pure compression systems, in particular for masonry structures [21]. At the same time, a coupled form-finding and grid optimization was proposed by Richardson et al. [22].

In the present paper, the shape of the vault model is principally obtained by a form finding method fine-tuned by one of the authors. In particular, the proposed method for the definition of the form is based on a multi-body rope approach (MRA) with masses connected by inextensible ropes characterized by a certain slack coefficient and by a certain degree of the constraint conditions [7]. This method appeared particularly adaptable in the case of free shapes with standardized constituting elements. One of the purposes of this work, in fact, is to propose an evaluation not only on the goodness of the form finding method adopted but also considering the best solution among the different pattern of the pierced configurations (disposition of the holes). The models and the stability evaluations are also performed in order to evaluate, given a starting configuration, the best and the worst evolution path for the increasing pierced area of the vault. These last results are of particular interest considering that the possibility to realize new holes and openings inside existing vaults it is more and more frequent in the refurbishment of existing large roofs originally built in the past. The models and the stability analysis were also performed in order to evaluate, for a given configuration, the best pattern for a step by step increasing of the pierced area in the vault.

Different funicular structures have been analysed in order to recognize the best shape together with the most efficient hole pattern configuration. The shape of the vaults is reached ranging in some chosen parameters such as the lowering degree (defined as the ratio between the maximum span $D$ and $h$, defined as the height of the vault), the slack coefficient of the original hanging net and the hole percentage $(H P)$ of the covering surface. The final shape and the pierced pattern were analysed by the TNA and the global structural stability analysis were made, through different load-cases and constrain conditions [22-25]. In addition, a scaled prototype of this vault typology is realized with the help of additive manufacturing process [26]. In Figure 1 the adopted procedure for the form-finding and the adopted span $(D)$ and the height $(h)$ are reported. The physical model was analysed by 3D Digital Image Correlation (DIC) technique under loading in order to recognize the pattern deformation. DIC technique, in particular, seems to be a very promising method to evaluate the stability and the collapse condition of these kinds of structures [24, 25]. The fast development of computer technology encouraged the increment of computational methods providing the basis for the diffusion of in vision-based full-field experimental measurements: e.g. 2D-DIC for SEM (scanning electron microscopy), AFM (atomic force microscopy) and planar loading and surfaces, 3D-DIC for general motion and deformation of curved or planar surfaces, V-DIC or Digital Volume Correlation for interior deformation measurements in opaque solids [24, 25]. Today, these approaches are used by researchers to find full-field quantitative measurement of motions and deformations. These kind of measurements have always been a significant issue in the assessment of mechanical properties, such as material strength or fracture parameters. Optical methods such as moiré interferometry [27], holography [28] and speckle interferometry [29] have been proven to be matured techniques to analyze macroscopic parameters and were used efficaciously in several different uses. However, all the interferometric techniques have severe prescriptions for the system's stability. The technical difficulties in the experimental realizations pushed many researchers' attention on the computerized procedures [30-32] in order to established automatic data acquisition from patterns.

Recently, a non-contacting optical method, digital image correlation (DIC), has been proposed by Sutton et al. [3137] and Bruck, et al. [31]. This method was applied to measure both strains and displacements in disparate conditions. The principal uses of the technique regarded microscopic strain measurements in electronic packaging [31], strain fields in polyurethane foam plastics and the estimation of their elastic parameters [32], without neglecting the evaluation of thermal effects in welded junctions. DIC was even used for in situ assessment of mural frescoes conservation [27]. The technique is based on two speckled frames, which were acquired by a solid state video camera, to reproduce the states of the object before and after the deformation. Using the concept of digitalization, it is possible to 


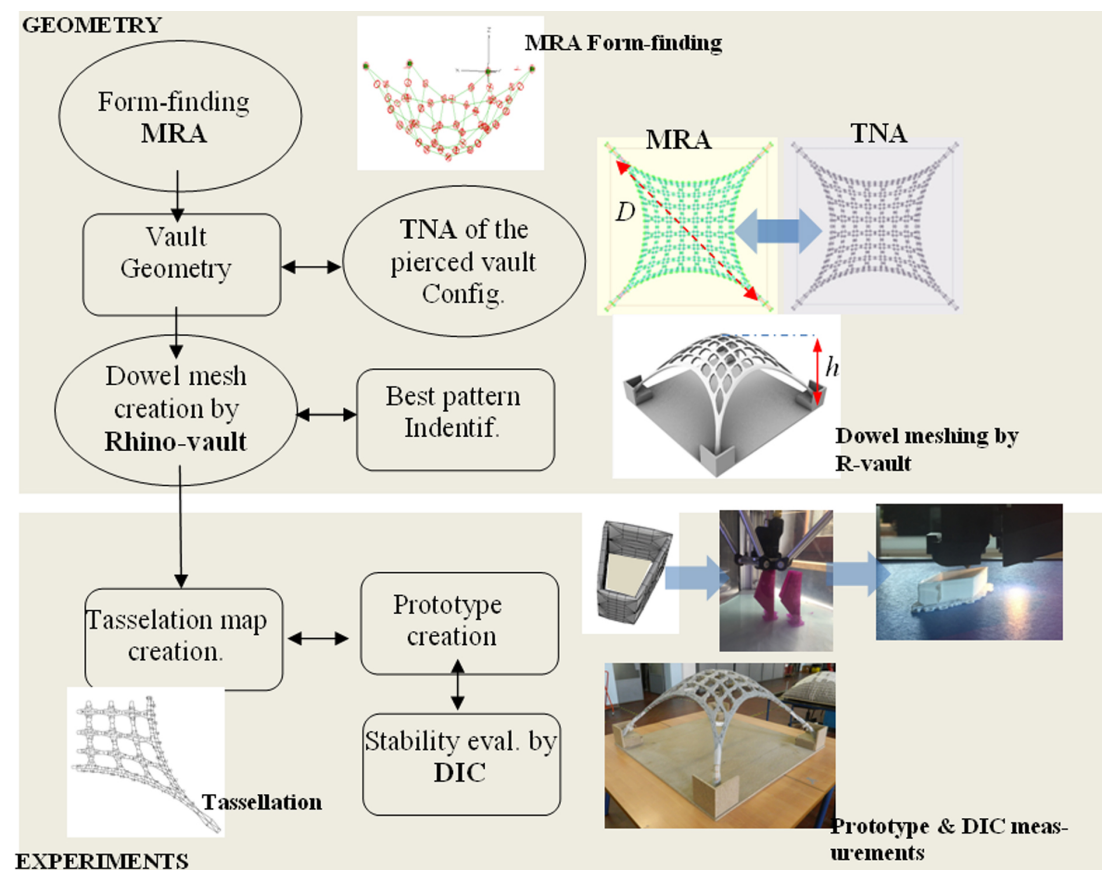

Figure 1: Flowchart of the Form-finding and the hole patterns definitions. The Form-finding is based on the MRA approach and is validated by the TNA. By the Rhino-vault application different patterns were considered and the tessellation map created. The scale model realized with a pierced area percentage of about $45 \%$ was analysed by DIC during loading tests

differentiate the pictures by the patterns levels and different intensity light levels. Both the two digitized images are then correlated by a specific algorithm, working on their mutual correlation coefficient or other statistical functions, to find out the refined dissimilarities between them. In the present paper, DIC is performed to distinguish typical deformation of the vault according to the loading condition. 3D-DIC measurements were used to recognize, preliminary, the critical condition of the structure before the collapse condition. As it will be shown in the following, these results can be obtained by the monitoring of specific portions (areas) at the extrados of the vault.

\section{Form-finding and hole patterns: The shape and the surface}

The first series of analysis were devoted to the form finding of different shapes. In this design phase, a squared geometry $\left(16 \times 16 \mathrm{~m}^{2}\right)$ for a free form compressed vault is chosen together with few main ranging parameters: the lowering degree $(D / h)$ and the hole percentage $(H P)$. The form-finding approach adopted in the paper was originally presented by one of the authors in 2004 [38] and was successively revised and expanded concerning to the original idea [7].
In particular, by MRA, it is possible to achieve disparate forms by the dynamic simulation of a hanging lattice constituted by spherical masses joined by flexible ropes falling down in the time domain [38]. In this case, any kind of loads can be used as input for the step-by-step procedure and both 2D and 3D systems may be investigated. Following this approach, named multi-bodies rope approach (MRA), it is possible to make the hanged mesh, for a precise group of masses, creating the final 3D funicular state [7]. In a standard MRA a generic node " $i$ " of a suspended net with quadrilateral mesh is examined. At this node a number $j$ of ropes (such as 4: $a, b, c, d$ ) are converging. At the same time, $s_{j}$ represents the forces inside them. The node " $i$ " is identified by the coordinates $x_{i}, y_{i}, z_{i}[7,38]$. The generic external load configuration is $p_{m}$, very often coincident with the self-weight simulated by the mass at each sphere. In the equation system, the inertial and the dissipative actions are taken proportional to the velocity and the acceleration of each node of the suspended grid. The equilibrium of the node $i$, is ensured by the following equation [7]:

$$
R_{i}=\sum_{j=1}^{n} s_{j}+\sum_{m=1}^{n 2} p_{m}+f^{I}+f^{I I}=0
$$

where $R_{i}$ was the resultant in the node $i$ at each step, $f^{I}$ was the inertial force with a module equal to the product between the mass of the node and the amplitude of the acceleration vector with a verse equal to the opposite verse of 
the acceleration itself. $f^{I I}$ was the dissipative force assumed equal to the product of a constant times the velocity vector with a direction equal to the opposite of the velocity itself. $s_{j}$ represented the forces in the ropes converging in the node $i$. The numbers $n$ and $n_{2}$ are, respectively, the number of the ropes converging in the node and the external loads applied on the same node [7, 38].

According to this approach, for the initial configuration of the grid nodes, a shape even very far from the final equilibrium step (configuration with minimum kinetic energy) can be assumed. The convergence of the system, in fact, was guaranteed by the convergence of the iterative process as a physical process of the three-dimensional suspended grid. In this case, the non-linear system of equations can be written for $\mathrm{z}, y$ and $z$ directions, is a system of non-linear differential equations to be solved by numerical methods [7, 37]. According to this frame, the solutions are found with the dynamic balance equations using a step-bystep analysis [7]. The contribution of the velocity and the acceleration, in $x$ direction, for each node (mass particle), can be expressed as [7, 38]:

$$
\begin{gathered}
\dot{x}_{i}=\frac{\partial x_{i}}{\partial t} ; \quad \dot{y}_{i}=\frac{\partial y_{i}}{\partial t} ; \quad \dot{z}_{i}=\frac{\partial z_{i}}{\partial t} \\
\ddot{x}_{i}=\frac{\partial^{2} x_{i}}{\partial t^{2}} ; \quad \ddot{y}_{i}=\frac{\partial^{2} y_{i}}{\partial t^{2}} ; \quad \ddot{z}_{i}=\frac{\partial^{2} z_{i}}{\partial t^{2}} .
\end{gathered}
$$

Similar equations can be written for $y$ and $z$ directions. Due to the large number of variables of the model, the author adopted a numerical approach to solve the system by a multi-bodies numerical code based on Runge-Kutta's solution method. Additionally, in the case in which a very large number of nodes are necessary in the form-finding process, a starting configuration obtained by NURBS (Non Uniform Rational Basis-Splines) surfaces can be used in the MRA to reduce the calculation time. It is clear that by MRA, it is possible to define the configuration of the structure as the reversed model corresponding to the final step (equilibrium step) of the hanging net [7]. The main peculiarity of the method here used (MRA) resides in the process of form-finding based on inextensible rope elements. The MRA method guarantees, in the final suspended form of the net, only traction elements also with very complex, (asymmetrical, horizontal loadings) loading configurations. This ensures only compressed elements in the overturned configuration (grid). Furthermore, with MRA, having to do with inextensible elements (ropes), it is possible to define, in advance, the length of the ropes that will be the beams in the reversed shape. In the present paper, taking into account that the form-finding was used to define compression-only vault optimal shapes the geometries obtained by the MRA were also validated by the TNA in order to obtain a confirmation of the funicular shapes. TNA has been used because of its intuitiveness and flexibility based on principles of the two-dimensional Graphic Static to solve the equilibrium of three-dimensional structures [21]. In this case, the validation of the shape preliminary obtained by MRA was performed by the TNA application realized with Rhino-vault, a free plug-in for Rhinoceros recently implemented by ETH Zurich [21].

The constraint conditions, as in the case of models realized by MRA, regard the four corner points and they are considered as fixed point on the ground supporting the entire vault. The maximum difference between the two kinds of solution of the final geometry obtained respectively by MRA and TNA were between $2 \%$ and $5 \%$.

For the pierced vault, one of the most important parameter is the amount of the holes (hole percentage: $H P$ ) and the positions of these openings (pattern). The authors considered these parameters key-points in the design of new masonry vaults and, particularly, in the refurbishment of existing ones. The enlarged necessity to increase the openings for natural light and the ever-present need to save material led the research to the study of pierced vault typology (see Figure 2). The analysis started fixing the ranging parameter. The vault heights equal to $2.5,5$ and 7.5 meters were investigated. At the same time different thickness of the vaults equal to $100,150,200,250,300 \mathrm{~mm}$ were considered in the analysis. Finally, as it can be observed in Figure 3, the hole positioning strategy is reported varying the four patterns. In particular, central-central (a), lateral-central (b), corner-diagonal (c) and central-corner (d) strategies were adopted to increase the number of holes $(H P)$ starting from an assumed initial shape.

For this reason, not only the number of the holes was evaluated but also the geometrical patterns and their spreading strategy in the growth of the pierced area. The different patterns have been divided into 4 families (groups), according to different paths for the increasing steps, corresponding to different number and different positions of the holes (see Figure 3). In Figure 3 the four patterns and the pierced area growth are reported together with the hole percentage, the number of holes adopted for each step and the positions for each case. For all the patterns considered the maximum pierced percentage is $45.46 \%$ and corresponds a number of 49 holes.

As previously reported, the purpose of the present paper was to compare the effect of an increasing percentage of the pierced areas for a given shape. Obviously, the form of the vault is certainly a function of the loading conditions applied during the form finding. Lower or higher $H P$ did not affect particularly the shapes obtained by MRA, espe- 


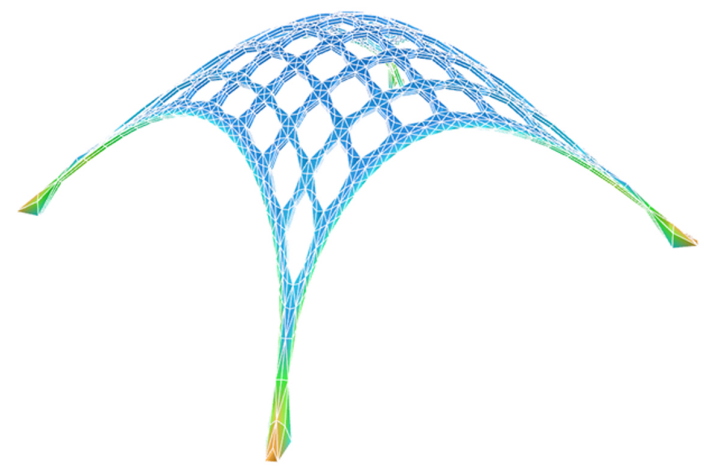

(a)

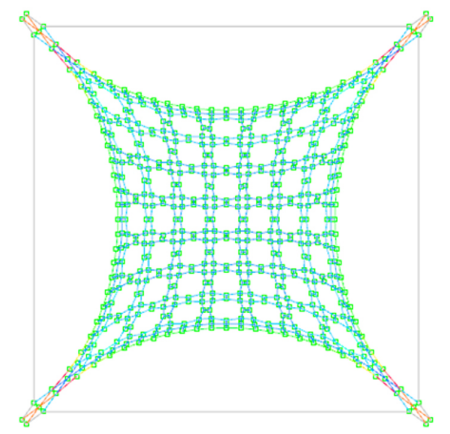

(b)

Figure 2: Configuration of the vault after forming with a maximum pierced area percentage of $45.46 \%$. Axonometric view (a). Top view (b)
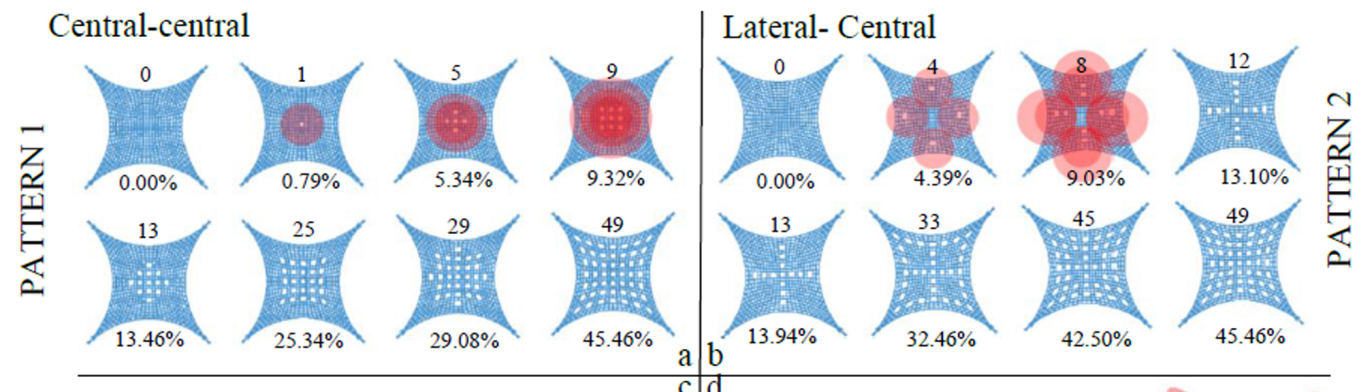

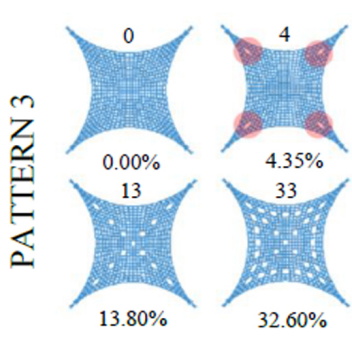

Corner-Diagonal

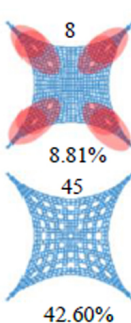

$42.60 \%$

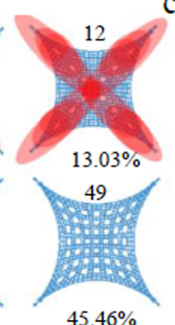

$45.46 \%$

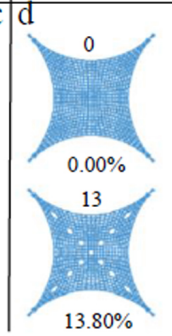

Central-Corner

Figure 3: The different patterns were divided into 4 families according to four different strategies of the positioning of the holes. Different paths for the increasing growth of the pierced areas on the vaults are used (red shaded indicators represented the four beginning steps for the four families). For each step the percentage of the subtracted area and the number of holes are indicated near the top view of the vault

cially comparing the extreme conditions ( $0 \%$ and $45.46 \%$ of pierced area). The suspended model, in fact, was made up of spherical masses connected each-other by inextensible ropes where the forming forces are concentrated in the nodes (barycentre of the spheres). Furthermore, the holes are positioned in correspondence to the empty spaces of the suspended net. At the same time, the reduced differences found between the shapes obtained with MRA and those obtained with the TNA allow to consider the two procedures, in this case, perfectly interchangeable. On the other hand, the analyses here reported are performed considering solely effects of height, thickness, $H P$ and propagation strategy of the holes for the four configuration types. A different approach might have considered further conditions, prin- cipally for intermediate steps, where at different positions (percentage and pattern strategy) of the holes may correspond significant effects in the final shapes. Anyway we focused, in the present context, numerical and experimental investigations exclusively on the parameters mentioned above. This choice was done to assess the importance that a certain $H P$ and its accretion strategy may impact existing shell spatial structures, not to mention the lowering degree and the slenderness of the structure.

Each geometrical solution has been verified by FEM analysis. In Table 1 the mechanical characteristics of the selected mortar are reported. The FEMs have been subjected before to static and after to buckling analysis to study the global stability of each structural configuration according 


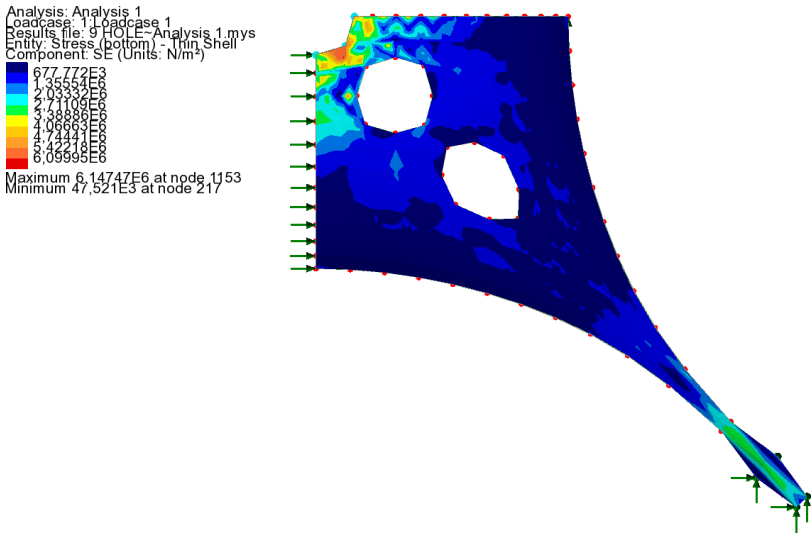

(a) Patter $a$, thickness $150 \mathrm{~mm}$, (Hole percentage 9.32\%

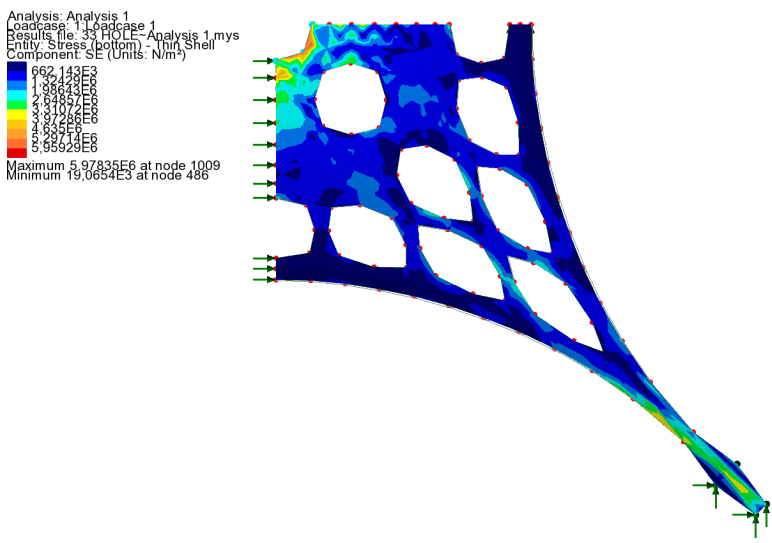

(c) Patter c, thickness $150 \mathrm{~mm}$, (Hole percentage $35.60 \%$
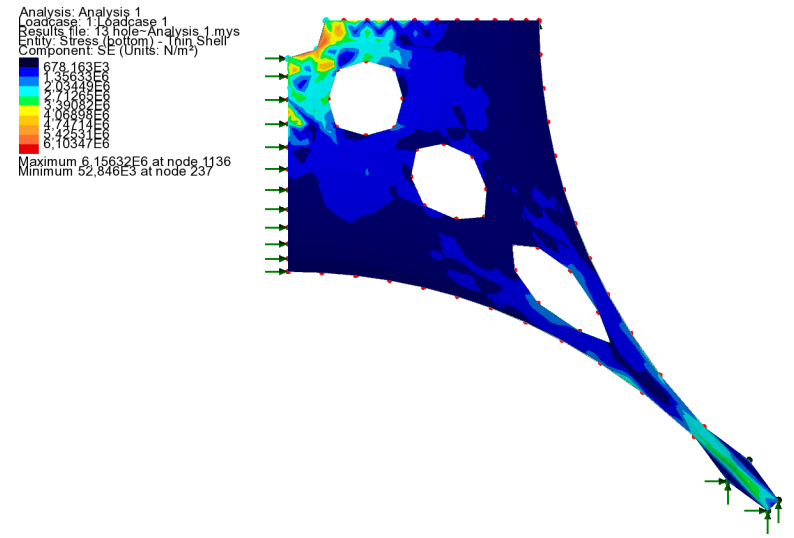

(b) Patter $a$, thickness $150 \mathrm{~mm}$, (Hole percentage 13.46\%
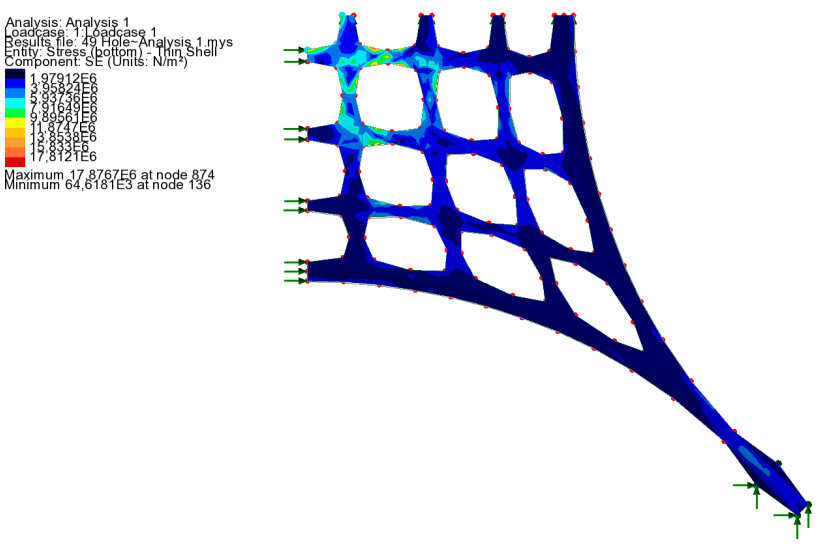

(d) Patter $c$, thickness $150 \mathrm{~mm}$, (Hole percentage 45.46\%

Figure 4: In this figure some of the most representative results in terms of stress field are reported. Von Mises stress plots for pattern $a$ are represented in the cases of hole percentages equal to $9.32 \%$ (a) and $13.46 \%$ (b) (steps with 9 and 13 holes). Similar results are reported for pattern $c$ in the cases of hole percentages equal to $35.60 \%$ (c) and $45.46 \%$ (d) (steps with 33 and 49 holes)

Table 1: Mechanical characteristics of the selected masonry

\begin{tabular}{cccccc}
\hline \multicolumn{5}{c}{ Vault material: Bricks and Cement Mortar } \\
\hline $\mathrm{f}_{m}$ & 6,5 & {$[\mathrm{MPa}]$} & $\mathrm{E}$ & 4550.0 & {$[\mathrm{MPa}]$} \\
$\tau_{0}$ & 0,28 & {$[\mathrm{MPa}]$} & $\mathrm{G}$ & 1137,5 & {$[\mathrm{MPa}]$} \\
$\gamma$ & 15 & {$\left[\mathrm{kN} / \mathrm{m}^{3}\right]$} & & & \\
\hline
\end{tabular}

to the varying parameters mentioned above [23-25]. The first critical load multiplier which corresponds to the first global and critical Eigen-mode of the structure has been found for each analysed case.

TSL6 finite element was selected for the structural analysis. It is a triangular element belonging to the group of shell elements useful for the simulation of curved shells, including multiple branched connections. The elements are able to simulate curved geometry with different thickness with anisotropic and composite material characteristics. The element type accounts for both membrane and flexural behavior. As expected by thin shell theory, transverse shearing deformations are omitted. In Table 2 the mechanical parameters of the selected material (masonry) implemented in the models are shown. The maximum stress under compression is assumed equal to $6.5 \mathrm{MPa}$. The maximum percentage of the pierced part for all the four families of vaults is $45.5 \%$ $(H P)$ corresponding to a number of holes equal to 49 . According to the values reported in Table 1 the simulations were conducted considering the proper load of the vault. As it can be seen from Table 2, all the von Mises maximum values, expressed in $\mathrm{MPa}$, have been reported in relation to different $H P$ and considering a varying $t$ for a lowering degree $D / h=3.2(h=7.5 \mathrm{~m})$. The data were reported for each pattern and for each increment of HP. In addition, as reported in Table 2 in the case of a vault without holes $(H P=0 \%)$, a thickness $(t)$ of $200 \mathrm{~mm}$ can be considered as a limit for the vault thickness. In fact, considering a further reduction to $t=150 \mathrm{~mm}$, the maximum stress values 
Table 2: Von Mises Stresses considering $H P$ accretion and vault thickness $(t) . D / h=3.2$

\begin{tabular}{|c|c|c|c|c|c|}
\hline Thickness & 300 & 250 & 200 & 150 & 100 \\
\hline Pattern, $\mathrm{N}$ of Holes $\mathrm{Hl}$ & \multicolumn{5}{|c|}{$\sigma_{d}[\mathrm{MPa}]$} \\
\hline a) $0(0.00 \%)$ & a) 3.52 & a) 3.87 & a) 5.89 & a) 10.07 & a) 21.03 \\
\hline b) $0(0.00 \%)$ & b) 3.52 & b) 3.87 & b) 5.89 & b) 10.07 & b) 21.03 \\
\hline c) $0(0.00 \%)$ & c) 3.52 & c) 3.87 & c) 5.89 & c) 10.07 & c) 21.03 \\
\hline d) $0(0.00 \%)$ & d) 3.52 & d) 3.87 & d) 5.89 & d) 10.07 & d) 21.03 \\
\hline a) $1(0.79 \%)$ & a) 1.94 & a) 2.36 & a) 2.96 & a) 4.83 & a) 9.68 \\
\hline b) $4(4.39 \%)$ & b) 2.87 & b) 4.00 & b) 5.98 & b) 9.99 & b) 20.27 \\
\hline b) $4(4.39 \%)$ & c) 3.42 & c) 4.84 & c) 7.39 & c) 12.69 & c) 26.77 \\
\hline d) $1(0.79 \%)$ & d) 1.94 & d) 2.36 & d) 2.96 & d) 4.83 & d) 9.68 \\
\hline a) $5(5.40 \%)$ & a) 2.53 & a) 3.43 & a) 4.94 & a) 7.89 & a) 15.61 \\
\hline b) $8(9.03 \%)$ & b) 3.58 & b) 4.92 & b) 7.46 & b) 12.69 & b) 26.56 \\
\hline b) $8(9.03 \%)$ & c) 3.43 & c) 3.85 & c) 7.41 & c) 12.73 & c) 26.89 \\
\hline d) $5(5.40 \%)$ & d) 2.53 & d) 3.43 & d) 4.94 & d) 7.89 & d) 15.61 \\
\hline a) $9(9.32 \%)$ & a) 2.06 & a) 2.75 & a) 3.92 & a) 6.15 & a) 11.80 \\
\hline b) $12(13.1 \%)$ & b) 7.60 & b) 10.53 & b) 15.60 & b) 25.33 & b) 49.65 \\
\hline c) 12 (13.1\%) & c) 2.92 & c) 4.03 & c) 5.94 & c) 9.66 & c) 18.59 \\
\hline d) $9(9.32 \%)$ & d) 2.06 & d) 2.75 & d) 3.92 & d) 6.15 & d) 11.80 \\
\hline a) $13(13.46 \%)$ & a) 2.00 & a) 2.69 & a) 3.86 & a) 6.16 & a) 11.87 \\
\hline b) 13 (13.94\%) & b) 3.85 & b) 5.27 & b) 7.70 & b) 12.45 & b) 24.15 \\
\hline c) $13(13.80 \%)$ & c) 2.00 & c) 2.69 & c) 3.86 & c) 6.16 & c) 11.87 \\
\hline d) $13(13.80 \%)$ & d) 2.00 & d) 2.69 & d) 3.86 & d) 6.16 & d) 11.87 \\
\hline a) $17(17.60 \%)$ & a) 5.61 & a) 7.49 & a) 10.53 & a) 16.02 & a) 27.85 \\
\hline b) 33 (32.46\%) & b) 3.84 & b) 5.25 & b) 7.69 & b) 12.52 & b) 24.60 \\
\hline c) $33(35.60 \%)$ & c) 2.13 & c) 2.61 & c) 3.73 & c) 5.98 & c) 11.72 \\
\hline d) $17(17.60 \%)$ & d) 5.61 & d) 7.49 & d) 10.53 & d) 16.02 & d) 27.85 \\
\hline a) $29(29.08 \%)$ & a) 3.71 & a) 5.05 & a) 7.30 & a) 11.61 & a) 22.56 \\
\hline b) 45 (42.50\%) & b) 4.52 & b) 5.86 & b) 8.04 & b) 12.03 & b) 23.66 \\
\hline c) $45(42.60 \%)$ & c) 2.93 & c) 3.91 & c) 5.57 & c) 8.79 & c) 16.46 \\
\hline d) $17(29.08 \%)$ & d) 3.71 & d) 5.05 & d) 7.30 & d) 11.61 & d) 22.56 \\
\hline a) $49(45.46 \%)$ & a) 5.36 & a) 7.38 & a) 10.89 & a) 17.88 & a) 35.44 \\
\hline b) 49 (45.46\%) & b) 5.36 & b) 7.38 & b) 10.89 & b) 17.88 & b) 35.44 \\
\hline c) $49(45.46 \%)$ & c) 5.36 & c) 7.38 & c) 10.89 & c) 17.88 & c) 35.44 \\
\hline d) 49 (45.46\%) & d) 5.36 & d) 7.38 & d) 10.89 & d) 17.88 & d) 35.44 \\
\hline
\end{tabular}

were over $10 \mathrm{MPa}$, very high respect to the limit of $6.50 \mathrm{MPa}$. Taking into account a maximum span $D$ equal to $2.4 \times 10^{3}$ $\mathrm{mm}$ (including supports). The slenderness $(\lambda)$ limit value for this type of configuration is $120(\lambda=D / t)$. When $H P$ increased up to $4.39 \%$, corresponding to 4 holes, pattern (b) appeared to be the best one, reaching a stress value just below to the maximum one for $t=150 \mathrm{~mm}$ and $\lambda$ equal to 160. It is important to note that the presence of central hole in the vault (pierced area percentage $=0.79$ ), as it was expected, involved a considerable reduction in the maximum stress level. In fact, for pattern $a$ and pattern $d$ the stress values dropped down to $1.94 \mathrm{MPa}$ with a decreasing of $55 \%$. On the other hand, if the pierced area percentage increases up to $13.1 \%$, the best group appeared to be $c$. Considering a pierced area percentage of $9.32 \%$, the best patterns were $a$ and $d$ with a maximum compression stress of $6.15 \mathrm{MPa}$ correspondent to $\lambda=160$. With an increase in the pierced area percentage over $30 \%$, the best pattern appeared to be pattern $c$ with a maximum stress value of $5.98 \mathrm{MPa}$ for $\lambda=160(t=150)$. Similarly, when the percentage raised above $40 \%$. In this case the pattern $c$ always appeared the best and the maximum stress is equal to $5.57 \mathrm{MPa}$. Finally, when the $H P$ reached $45.46 \%$ (maximum percentage) all patterns are equivalent and the only compatible $\lambda$ value was equal to $80(t=300 \mathrm{~mm})$. In Figure 4 some of the most representative results in terms of stress field are reported. 
Von Mises stress contour-plots for pattern $a$ are represented in the cases of hole percentages equal to $9.32 \%$ (a) and $13.46 \%$ (b) (steps with 9 and 13 holes). Similar results are reported for pattern $c$ in the cases of hole percentages equal to $35.60 \%$ (c) and $45.46 \%$ (d) (steps with 33 and 49 holes).

\section{Stability evaluation of the pierced vaults}

For the following analysis the configuration with $H P$ equal to $45.46 \%$ is considered for the evaluation of the most critical conditions according to the results reported in Table 2 . This configuration is represented in Figure $5 \mathrm{a}$ and $5 \mathrm{~b}$. For the evaluation of buckling analysis of this kind of structures, it is well known that the elastic equilibrium stability problem can always be written as [18]:

$$
\left\{[K]-\mu\left[K_{g}\right]\right\}\{\delta\}=\{0\}
$$

where $[K]$ is the elastic stiffness matrix, $\left[K_{g}\right]$ is the geometric stiffness matrix, $\{\delta\}$ is the nodal displacement vector and $\mu$ is the load multiplier. The eigen-values of the prob-

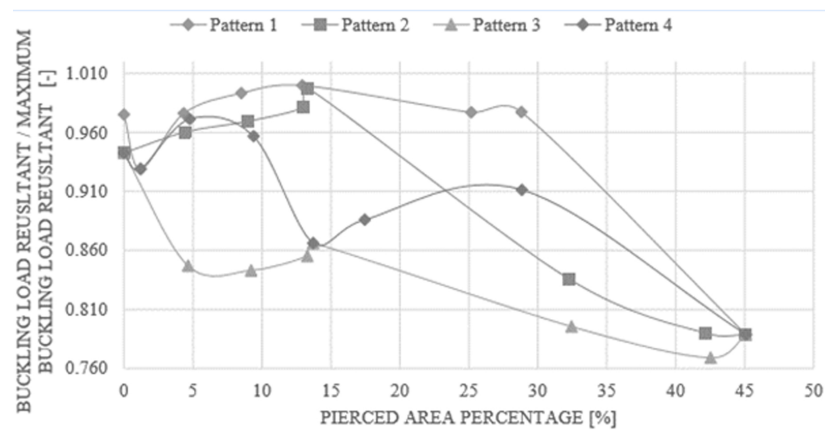

(a)

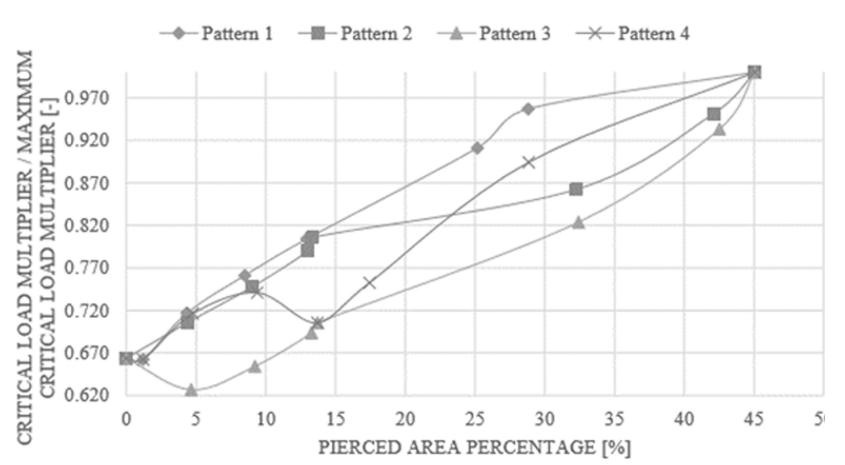

(b)

Figure 5: Normalized Buckling load for the four pattern evolutions considering the variation of the pierced area (a). Normalized critical load multiplier vs. pierced area percentage for the four pattern evolution (b) lem can be found from the condition $\operatorname{Det}\left([K]-\mu\left[K_{g}\right]\right)=0$. As the vault for the maximum pierced area is concerned, the critical loads multiplier evaluated for the structure is 2.71. In Figure 5a the normalized buckling load resultant vs. the pierced area percentage is reported for all patterns. In Figure $5 \mathrm{a}$ and $\mathrm{b}$ the case with $\lambda=80(\mathrm{~s}=300 \mathrm{~mm})$ and height equal to 7.5 is considered $(D / h=3.2)$. In Figure $5 b$ the normalized critical load multiplier vs. the pierced area percentage is reported for the same slenderness and lowering degree. Each function is representative of one of the four different patterns. In every analyzed case, when a solution with a larger pierced area is considered and characterized by an increment of the number of central hole, these solutions are always more stable in comparison to the case of a lower pierced area percentage. As already known, a concentration of openings in the top area of the vault generated more stable solutions. In general, roofs with the same percentage of pierced area are more stable increasing the lowering degree.

Taking into account that the obtained results are referred to a limited but quite extended analyzed number of cases (720 simulations), and considering that no evolutionary algorithm has been implemented for the exploration of the solutions (optimization), we summarized the results

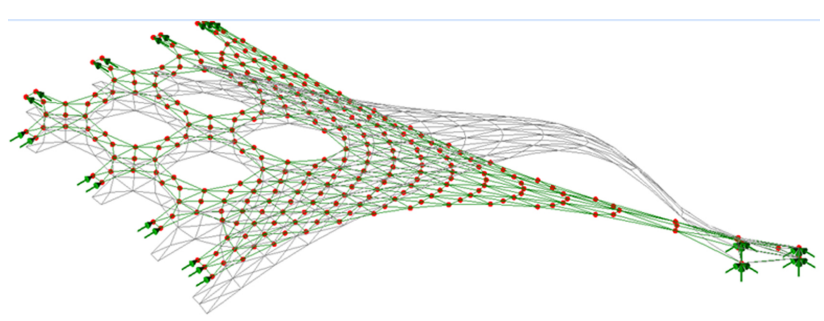

(a)

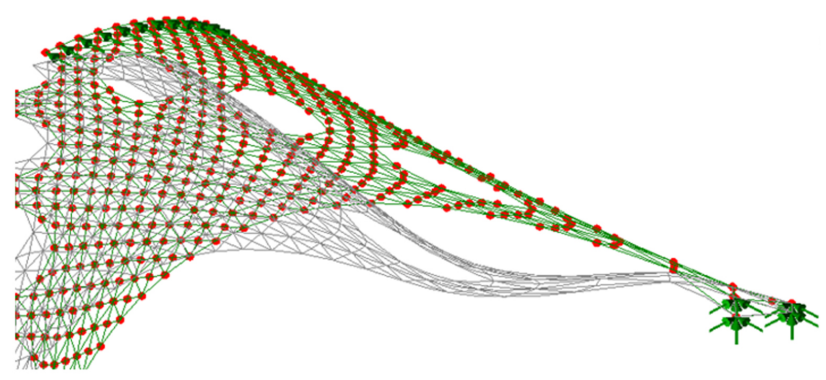

(b)

Figure 6: In this figure the collapse modalities of the structure are reported. The first modality "A" represented by far the most frequent one. In this case the cusp of the vault fell down, while the parts near the supports deformed by moving upwards (a). On the other hand, the second collapse modality in reported as the type "B". It is less frequent, as reported in Figure 5 . In this case the cusp of the vault fell down and the parts near the supports deformed by moving downwards (b) 


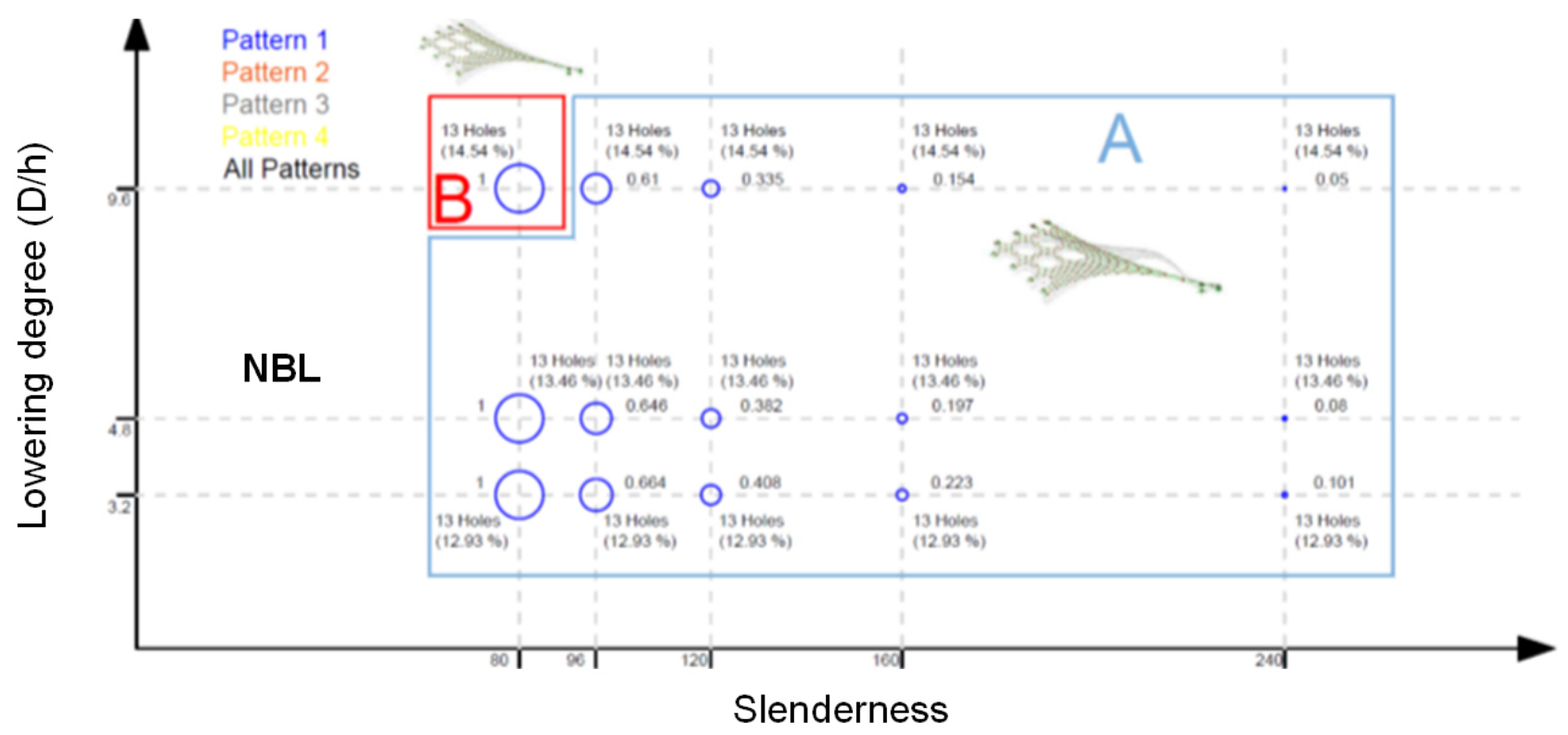

(a)

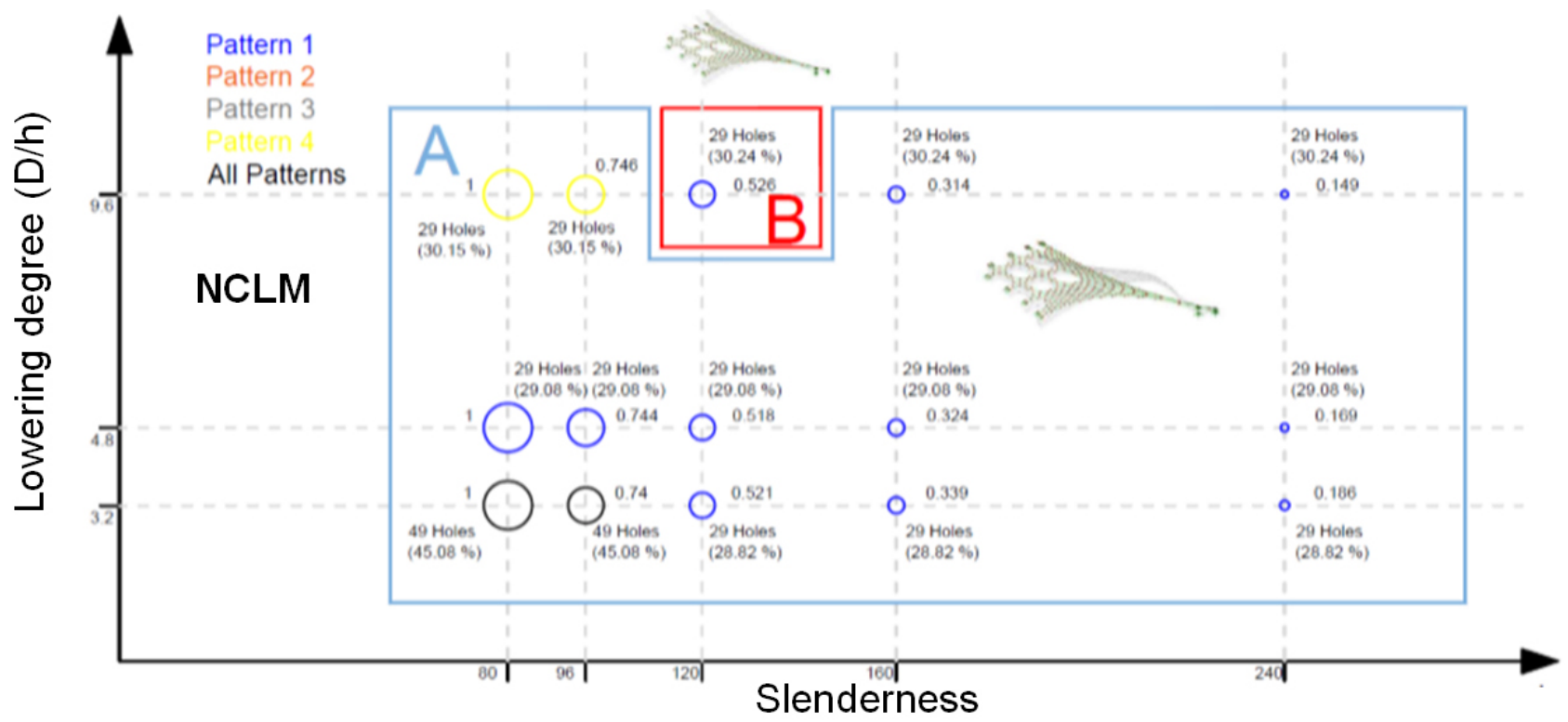

(b)

Figure 7: Normalized buckling load value (NBL), as regards the $D / h$ (ranging from 3.2 to 9.0 ) and the slenderness $(80<\lambda<240)$ are reported. For each value shown in the graph and represented by a circle, with color corresponding to the best pattern, the size of the indicator (circle) is proportional to the normalized value of the corresponding NBL (a). Normalized critical load multiplier (NCLM), as regards the $D / h$ and the slenderness are reported. For each value shown in the graph and represented by a circle, with color corresponding to the best pattern, the size of the indicator (circle) is proportional to the normalized value of the corresponding NCLM (b)

obtained in two schemes where the more stable solutions are identified according to the lowering degree and the slenderness of each particular geometrical configuration.

In Figure 6a the most frequent collapse mechanism (A) is reported. It is characterized by the bulging of the vault diagonal portions and the lowering of the cap. Only in one case the observed collapse mechanism is characterized by a different mechanisms noticed as the second type (B). It is characterized by the internal bulging of the diagonal parts of the vault and the raising of the cap (see Figure $6 \mathrm{~b}$ ). This last case (B) occurred for a slenderness equal to 80 and a very high $D / h(9.0)$.

In Figure 7a the best solutions in terms of stability conditions (Normalized buckling load value), as regards the 


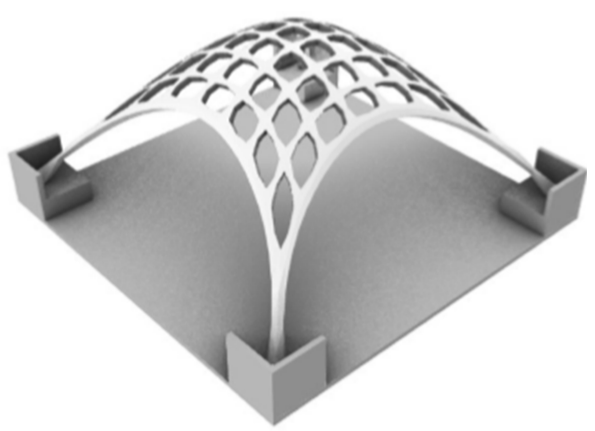

Figure 8: 3D Geometrical Model of the scaled prototype - Perspective View

$D$ over $h$ ratio (ranging from 3.2 to 9.0) and the slenderness level (from 80 up to 240) are reported. For each value shown in the graph and represented by a circle, with color corresponding to the best pattern, the size of the indicator (circle) is proportional to the normalized value of the corresponding normalized buckling load. In Figure $7 \mathrm{~b}$ a similar graph is reported, in this case the reference parameter is the critical load multiplier. Also in this case it is possible to identify a unique case where the B type collapse mechanism prevailed. This last condition is characterized by $\lambda=80$ and $D / h=9.0$ (pattern 1$)$.

\section{Experimental tests: The model of the vault}

The vault selected for the experimental validation presented the higher value of the critical load multiplier between the structures with a $D / h$ equal to 3.2. This model is characterized by 49 holes and a $H P$ of $45.5 \%$. It is 7.5 meters tall and the thickness of the transverse section is equal to $30 \mathrm{~cm}$. The scaled model used was realized with a reduction using a scaling factor of $0.0625(1 / 16)$ in order to have the side equal to $1 \mathrm{~m}$. Consequently, the prototype was $469 \mathrm{~mm}$ tall with a transverse section of $18.75 \mathrm{~mm}$ and slenderness equal to 80 (see Figure 8). Considering the double symmetry of the geometry, only a quarter of the vault is considered for the voussoir geometry design (see Figure 9). In the preliminary tests described in the present paper the primary objective was to analyze the global behavior of the structure under loading. In the next experiments possible effects of the dimensional analysis will be considered [40].

Using rapid prototyping toolkits, the shape of the vault was separated in several dowels. The design of the bricks consider that the discretized form diagram achieved in the form finding process shall be viewed as the forces in the
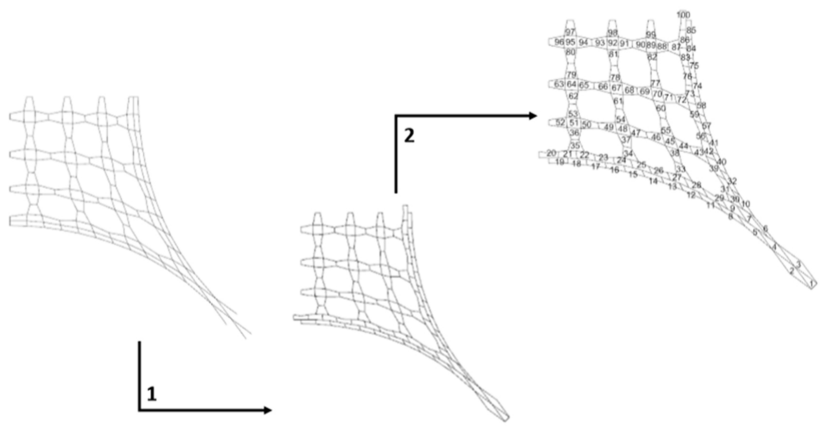

Figure 9: Bricks design process scheme - starting from the equilibrated form diagram output of the TNA, the bi-dimensional geometry of the bricks has been determined

vault when subjected to vertical loads. The bricks have to be realized in mortar cement so a mould system has to be designed. Due to the small scale of the model, it is very important to ensure the accuracy of the formwork geometry. The geometry of each formwork has been created into Rhinoceros using Grasshopper. Exporting the geometry source file these elements were realized with 3D printing with a Fuse Deposition Modeling (FDM) technique in PLA material (completely eco-friendly).

The most relevant parameters set up for the 3D printing process are: a filament diameter of $1.75 \mathrm{~mm}$ a layer height: $0.2 \mathrm{~mm}$, a fill density of $30 \%$ (see Figure 10). At the same time the print speed was between $80-150 \mathrm{~mm} / \mathrm{s}$ with a printing temperature of $215^{\circ} \mathrm{C}$.

It is remarked that for the sake of simplicity the four base elements have been fabricated with the FDM technique. All bricks were cast into the shuttering and made by cement mortar with a 0.4 water to cement ratio.

The three-dimensional shape of the 100 different bricks typologies and their formworks have been designed according to the previous scheme for a total number of 400 bricks composing the vault. To support the bricks during the prototype realization, a spar was realized with cardboards sheets designed parametrically with Grasshopper and cut on purpose according to the internal shape of the vault. The spar has been placed on a plywood panel supported by 4 threaded bars that allow to control the height of the whole structure during all the construction stages. According to the brick scheme designed previously, all the bricks have been placed on the spar upper surface. At the end of construction, the spar has been removed. During first down-lifting phases, the self-weight of the structure start to be carried out on the structure (Figure 10). 


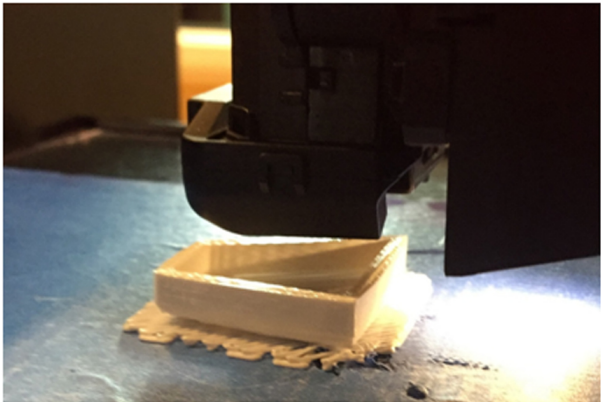

(a)

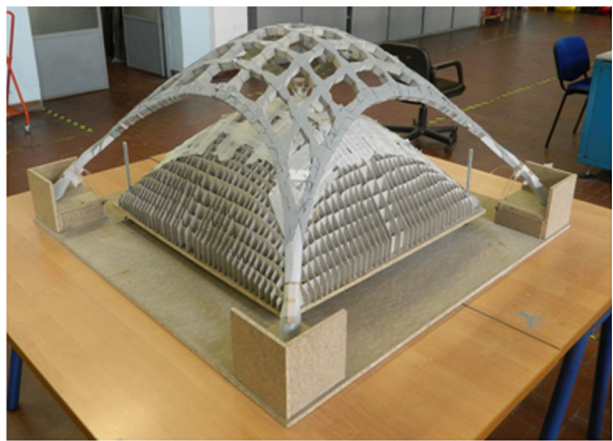

(c)

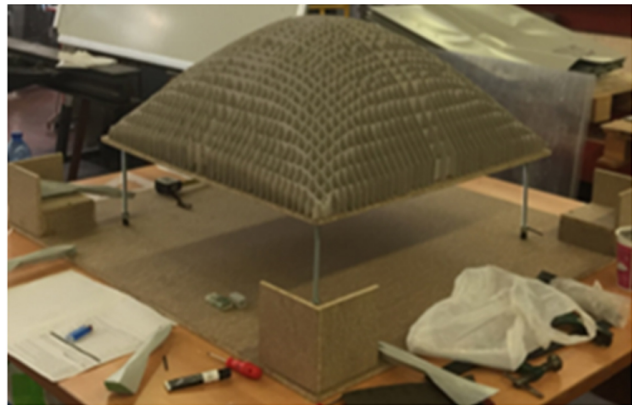

(b)

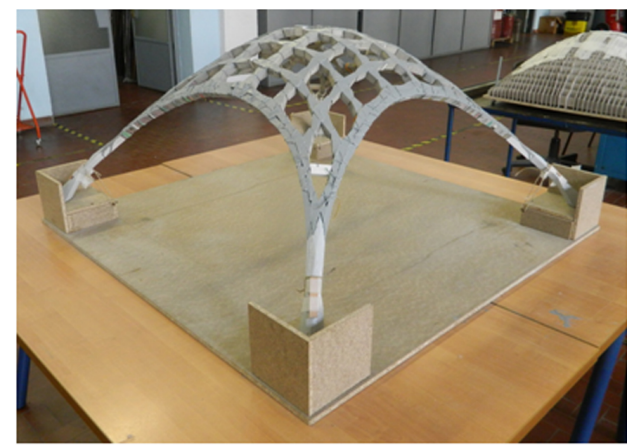

(d)

Figure 10: 3D printing with FDM of the formwork (a). To support the bricks during the prototype realization, a spar were realized with cardboard (b) Removing phase of the spar (c). The structure is realized (d)

\section{Stability analysis by digital image correlation (DIC): Preliminary results}

In order to analyze the vault model deformation under loading 3D-Digital Image Correlation (DIC) was used (Figure 11). The correlation algorithm used is based on the tracking of grey value pattern in small local neighborhoods. $\mathrm{G}(x, y)$ is the grey value of a pixel with the coordinate $\mathrm{x}$ and $\mathrm{y}$ inside of the subset, the correlation algorithm minimized the sum:

$$
\sum_{x, y}\left(G_{t}\left(x_{t}, y_{t}\right)-G(x, y)\right)^{2}
$$

where $G_{t}\left(x_{t}, y_{t}\right)=g_{0}+g_{1} G\left(x_{t}, y_{t}\right) ; x_{1}=a_{0}+a_{1} x+a_{2} y+a_{3} x y$ and $y_{1}=a_{4}+a_{5} x+a_{6} y+a_{7} x y$. By the variation of the illumination parameters $\left(g_{0}, g_{1}\right)$ and the parameters of the affine transformation $\left(a_{0} \ldots a_{7}\right)$ an accuracy for the matching of better than 0.01 pixel can be achieved. The correlation algorithm which is actually applied is a two dimensional generalization of the case just described. It implies, in particular, a two dimensional geometric transformation which is to rectify perspective distortion and relief displacement. In view of the small size of the image areas it is sufficient to apply affine linear transformation (six a type parameters). Once the 3D contour has been determined, the second step in digital 3D correlation is the measurement and the determination of the three-dimensional deformation of the object surface (deformed pattern). This process is carried out by correlation of the images, taken by both cameras with respect to their original reference images. In Figure 12a and Figure 13a DIC applications are shown together with the experimental set-up. In Figure $12 \mathrm{~b}$ and $13 \mathrm{~b}$ the load applications are reported. In particular, a total load of 88.30 $\mathrm{N}$ was applied in the structure by 4 successive steps. In Figure 13a the measurement areas are reported. In Figure 13b the last loading phase is shown.

The surfaces considered for the DIC measurements are not loaded by external loads (see Figure 13). This choice was made to avoid measurements by possible local effects that could take place on dowels constituting the vault where the loads were placed. In this way, the detection procedure can be transferred from the prototype scale to the structural scale. In fact, it appeared of great importance to explore a non-contact monitoring system that is able to interpret, through the observation of points localized on the external surface of the vault, or limited areas of the structure, the global collapse condition. Furthermore, the analysis of the 


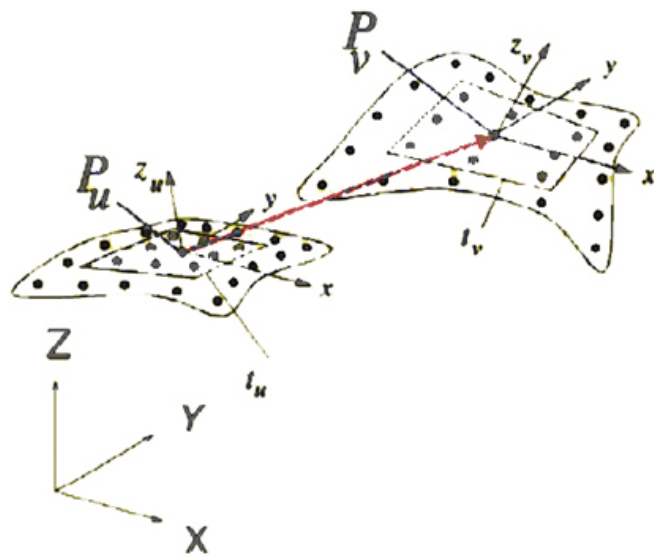

(a)

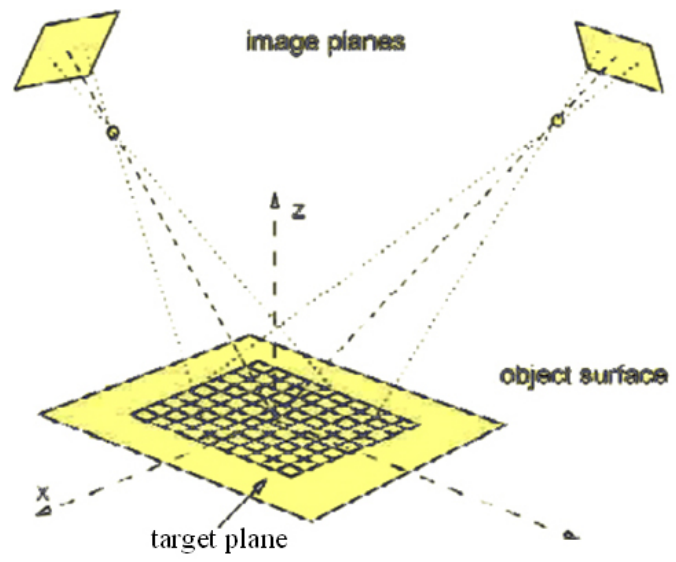

(b)

Figure 11: Determination of the three-dimensional displacement vector (a). Principle of 3D image correlation with 2 cameras (b)

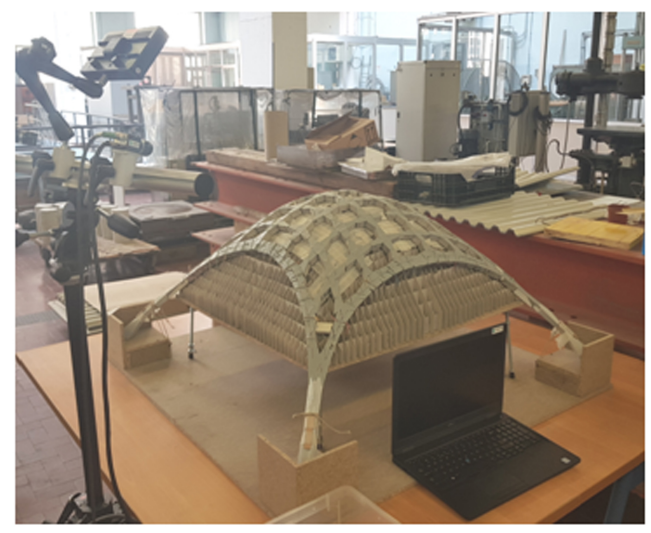

(a)

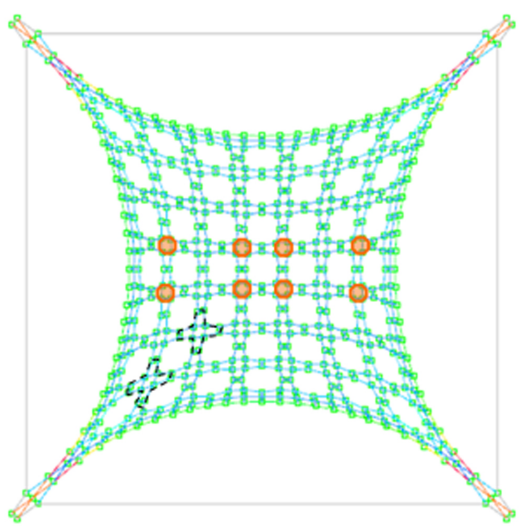

(b)

$$
\begin{aligned}
& \text { O Load applications } \\
& \text {, DIC measurement areas }
\end{aligned}
$$

Figure 12: DIC application is shown together with the experimental set-up (a). The geometry of the vault prototype is reported together with the points where the load applications were applied (b)

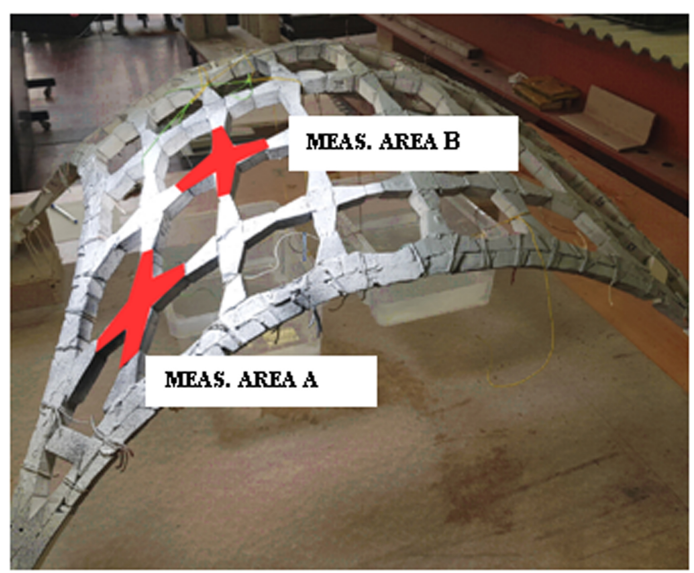

(a)

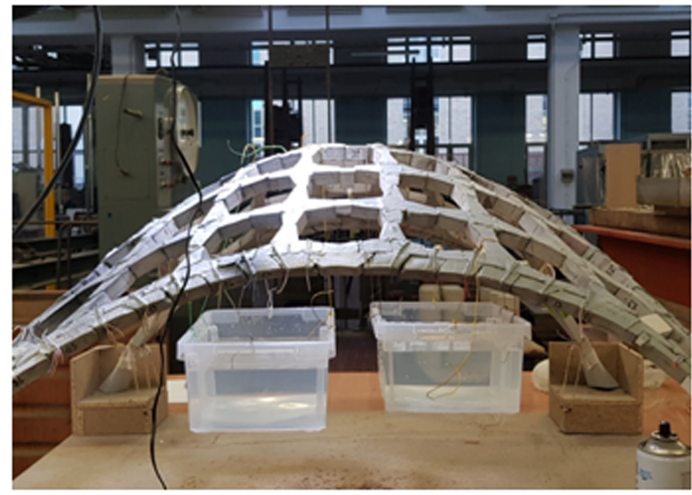

(b)

Figure 13: Physical model of the vault. In red the portion of the structure monitored by DIC (a). The loading procedure is obtained by different loads applied on the lower part of the structure during different loading steps realized by different water containers (b) 


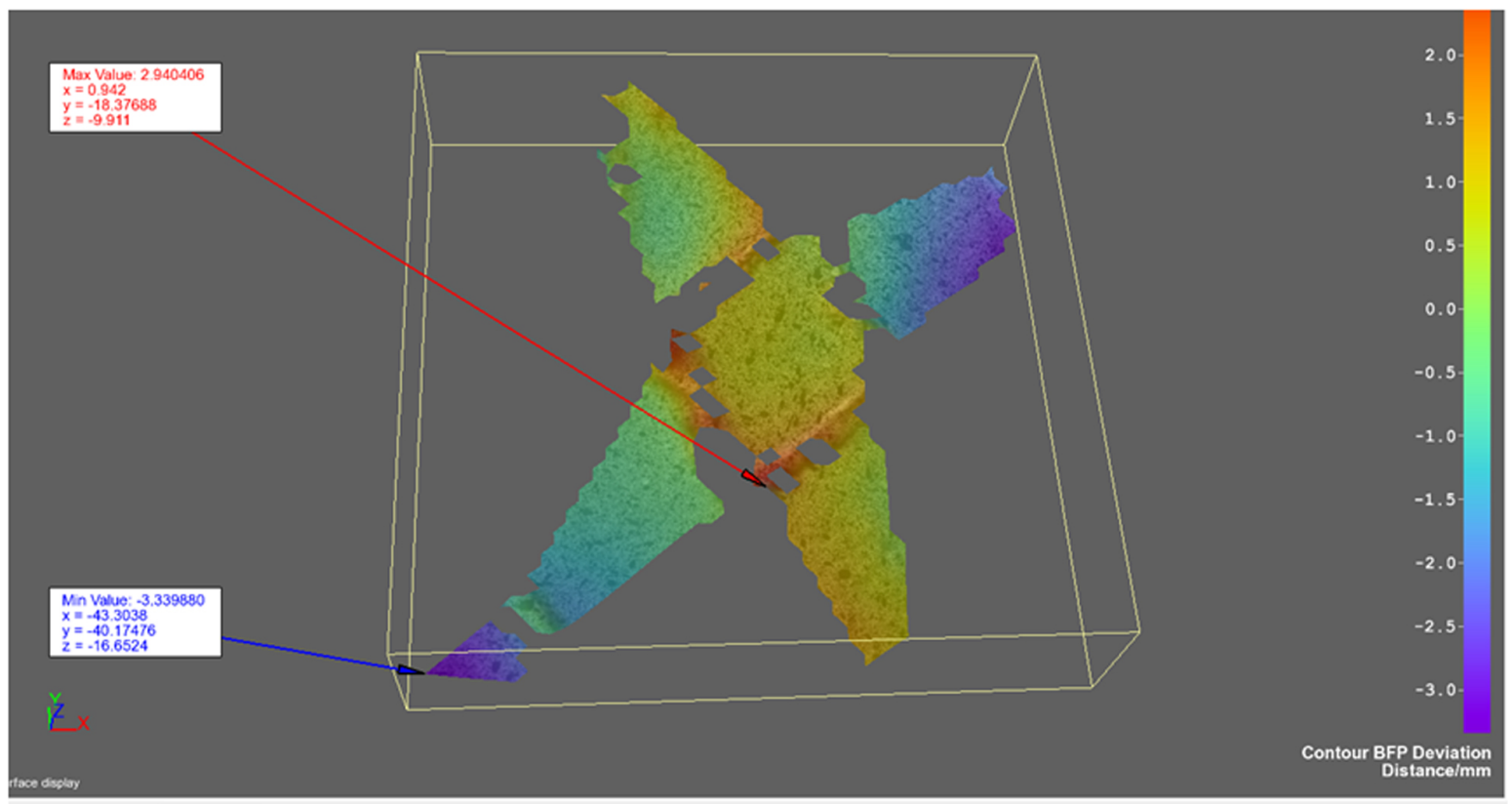

Figure 14: Strain Diagram for measurements 2. The minimum and the maximum values are reported

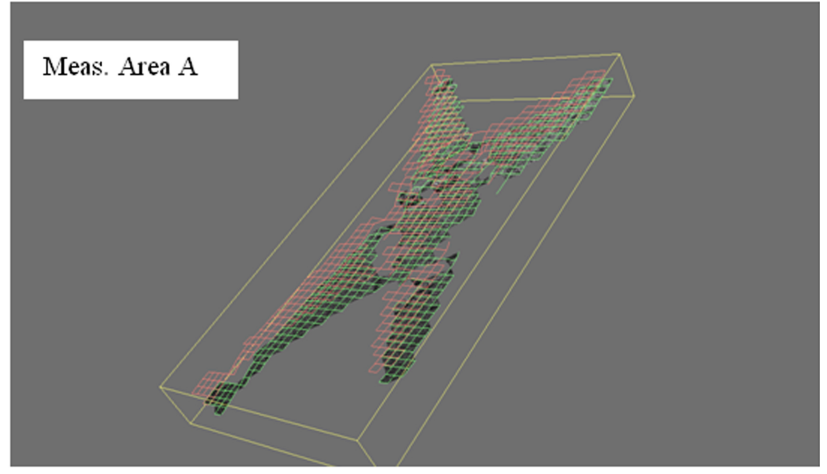

(a)

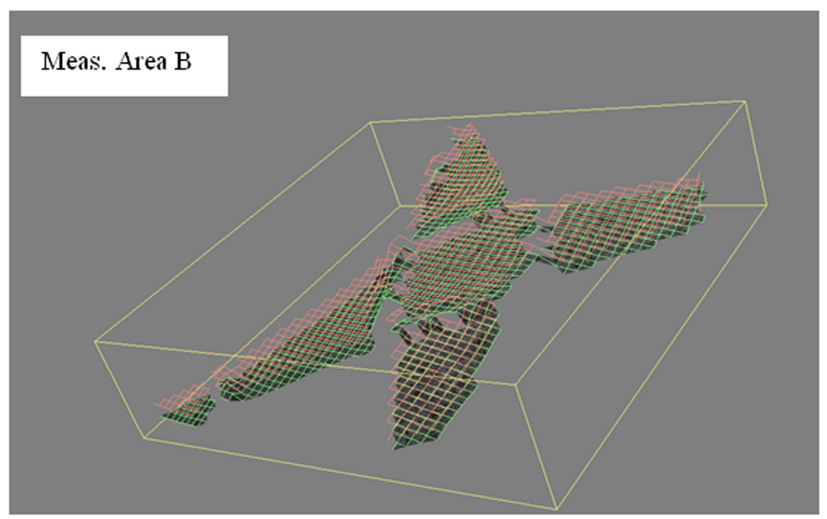

(b)

Figure 15: Deformed grids referred to the first and second measurement area surface portions, as shown in Figure 13, are more simply accessible, even in full-scale structures, if positioned in portions of the vault localized near the supports and not on the vault top, very difficult to be monitored in large vaults and existing roofs. In the Figures 14 and 15 the maximum and the minimum surface displacements for each of the two measurement areas are reported as the applied loads changed. It can be always observed an increasing of displacements of all the detected points up to the load phase just before the collapse condition of the model. The collapse phase of the prototype, in order to be able to carry out more tests on the same model, did not involve the complete collapse of the prototype, but the maximum value of the load was assumed in correspondence of the first undermining phenomenon of one of the dowels constituting the vault. Once this value was reached, the load increments were interrupted and the structure was unloaded. From the point of view of the detection of the maximum and the minimum displacements, this condition of "first damage" to the vault has always been accompanied by an evident reduction in the increase of the displacement measurements. As reported in Figure 16, the evolution of the displacement measurements as the load increased can be represented by a fourth degree polynomial displacement. The trend of the displacements can be expressed as a function of the imposed load: $d p_{4}(L)$. La stationarity of the function $d p_{4} / d L$ denounced the imminent condition corresponding to the collapse of the vault. 


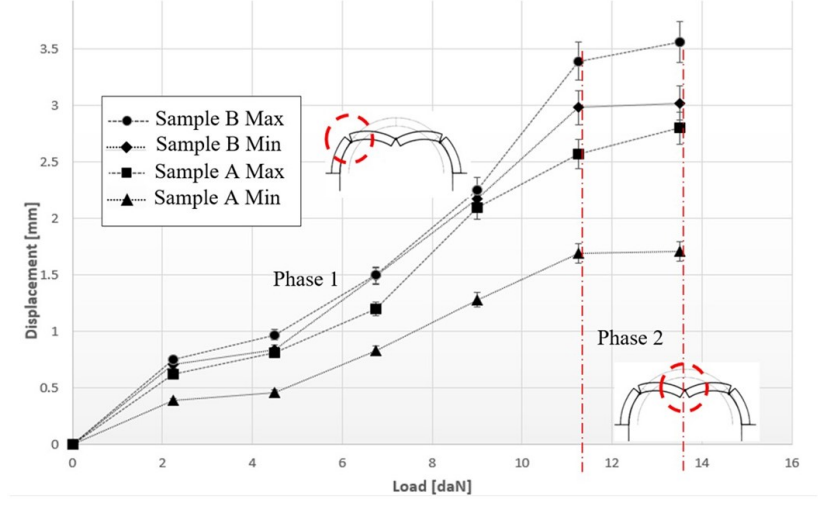

Figure 16: The trends related to the minimum and the maximum displacement measured in grids $A$ and $B$ are reported. In particular, two different phases are recognized, phase 1 and phase 2 (imminent collapse condition). La stationarity of the function $d p_{4} / d L$ denounced the imminent condition corresponding to the collapse of the vault.

\section{Conclusions}

In the present paper, the form finding process for a cement based tessellated pierced vault is proposed by MRA approach. The validity of the optimal shape of the compression-only vault was checked by the truss network analysis (TNA). At the same time, different tessellation meshes of the adopted vault shapes, according to different $H P$ and different strategies in the pierced area growth were investigated. In particular, central-central, lateral-central, corner diagonal and central-corner strategies were adopted to increase the number of holes.

The different solutions in term of $H P$ and strategy of the pierced area growth were compared in term of strength to the maximum stress under compression, assumed for the considered material equal to $6.5 \mathrm{MPa}$. The maximum percentage of the pierced part for all the four families (groups) of vaults was $45.5 \%$ corresponding to 49 holes. In Table 2 von Mises stresses in the material, considering different $H P$ steps (HP steps $=0.79 \%$ or $4.39 \%, 5.40 \%$ or $9.03 \%, 9.32 \%$ or $13.1 \%, 13.46 \%$ or $13.80 \%, 17.60 \%$ or $32.46 \%, 29.08 \%$ or $42.50 \%, 45.46 \%$ ) were summarized for all the analyzed configuration taking into account the different positions of the holes (pattern configurations) and slenderness ( $\lambda$ ranged from 80 to 240). In addition, taking into account all the configurations, the geometries of the vaults were analyzed by mean of global stability analysis identifying the best solutions according to different slenderness and a lowering degree $D / h=3.2$. Taking into account the results reported in Table 2 the best solution in term of $H P, \lambda$ and thickness $(t)$ was the case with 33 holes, $35.60 \%$ of pierced area, $t=$ $150 \mathrm{~mm}$ and characterized by a $\lambda=160$.
Furthermore, a 3D printing with a Fuse Deposition Modeling (FDM) technique in polylactide (PLA) material (completely eco-friendly) is explored for the construction of the formworks of the cement based blocks (dowels) useful for the assembly of a vault scaled prototype. The prototype of the vault, characterized by the maximum piercing percentage (45.5\%) was subjected to static loading conditions and monitored by a non-contact device based on the Digital Image Correlation (DIC) technique. The 3D-DIC was performed to recognize the structural behavior during the loading process of the model (prototype). In recent works it has been observed how the variation of some structural parameters, such as the natural frequency of the monitored elements, can offer information on the proximity to the collapse due to instability phenomena [41-43], without neglecting the stiffness reduction in the joints of truss structures [44]. In the present paper, the evolution of the displacement measurements as the load increased can be represented by a fourth degree polynomial displacement trend as a function of the imposed load: $d p_{4}(L)$. Stationarity of the function $d p_{4} / d L$ denounced the imminent condition corresponding to the collapse of the vault.

Acknowledgement: The MastrLab Laboratory of the Politecnico di Torino is gratefully acknowledged for the support in the experiments. Dr. F. Bazzucchi is also acknowledged for the numerical analysis. The MONFRON site Laboratory (Innovative Experimental Laboratory for the Multi-parameter Analysis of Safety and Structural Integrity of Brittle Material, https://monfronsitelab.wixsite.com/homepage) is gratefully acknowledged for DIC measurements performed during the tests.

Funding information: The authors state no funding involved.

Author contributions: All authors have accepted responsibility for the entire content of this manuscript and approved its submission.

Conflict of interest: The authors state no conflict of interest.

\section{References}

[1] Rippmann M, Lachauer L, Block P. Interactive vault design. Int J Space Structures. 2012;27(4):219-30.

[2] Oxman R, Oxman R. The New Structuralism: Design, Engineering and Architectural Technologies. New York: John Wiley; 2010. 
[3] Tessmann 0. Collaborative Design Procedures for Architects and Engineers. PhD thesis. University of Kassel; 2008.

[4] Ferguson ES. The Mind's Eye: Nonverbal Thought in Technology. Science. 1977;197(4306):827-36.

[5] Kilian A. Design exploration through bidirectional modeling of constraints. PhD thesis. Massachusetts Institute of Technology. Cambridge, MA; 2006.

[6] Tornabene F, Viola E. Static analysis of functionally graded doubly-curved shells and panels of revolution. Meccanica. 2013 May;48(4):901-30.

[7] Manuello Bertetto A. Multi-body rope approach for grid shells: form-finding and imperfection sensitivity. Eng Struct. 2020;221:111029.

[8] Burkhardt B, Bächer M. Multihalle Mannheim, Institute for Lightweight Structures (IL), 13, University of Stuttgart; 1978.

[9] Regalo ML, Gabriele S, Salerno G, Varano V. Numerical methods for post-formed timber grid-shells: simulation of the forming process and assessment of R-Funicularity. Eng Struct. 2020;206:110-9.

[10] Chilton J. The Engineer's Contribution to Contemporary Architecture: Heinz Isler. London: Thomas Telford Press; 2000.

[11] Killian A, Ochsendorf J. Particle-spring system for structural form finding. J Int Assoc Shell Spat Struct. 2005;45(147).

[12] Kuijvenhoven M, Hoogenboom PC. Particle-spring method for form-finding grid shell structures consisting of flexible members. J Int Assoc Shell Spat Struct. 2012;53(1):171.

[13] Pedersen P. Optimal joint position for space structures. J Struct Div. 1973;99(12):2459-76.

[14] Gil L, Andreu A. Shape and cross section optimization of a truss structure. Comput Struc. 2001;79(7):681-9.

[15] Becker M, Fleury C. A primal dual approach in truss typology optimization. Comput Struc. 1997;64(1-4):77-88.

[16] Giger M, Ermanni P. Evolutionary truss topology optimization using a graph based parametrization concept. Truct. Multidisc. Optim. 2006;32(4):313-26.

[17] Lamberti L. An efficient simulated annealing algorithm for design optimization od truss structures. Comput Struc. 2008;86(1920):1936-53.

[18] Rasmussen MH, Stolpe M. Global optimization of discrete truss topology desicn problems using a parallel cut-an-branch method. Comput Struct. 2008;86:1527-1538.

[19] Togan V, Daloglu AT. Optimization of 3D trusses with adaptive approach genetic algorithm. Eng Stuctures. 2006;28(7):1019-27.

[20] Winslow P, Pellegrino S, Sharma SB. Multi-objective optimization of free-form grid structures. Struct Multidiscipl Optim. 2010;40(16):257-69.

[21] Block P, Ochsendorf J. Trust network analysis: A new methodology for three dimensional equilibrium. J Int Assoc Shell Spat Struct. 2007;48(155):145-53.

[22] Richardson JN, Adriaenssens S, Coelho RF, Bouillard P. Coupled Form Finding and Grid Optimization Approach for Single Layer Grid Shells. Eng Struct. 2013;52:230-9.

[23] Carpinteri A, Bazzucchi F, Manuello A. Nonlinear instability analysis of long-span roofing structures: the case-study of Porta Susa railway-station. Eng Struct. 2016;110:48-58.

[24] Bazzucchi F, Manuello A, Carpinteri A. Instability load evaluation of shallow imperfection-sensitive structures by form and interaction parameters. Eur J Mech A, Solids. 2017;66:201-11.
[25] Bazzucchi F, Manuello A, Carpinteri A. Interaction between snapthrough and Eulerian instability in shallow structures. Int J Nonlinear Mech. 2017;88:88.

[26] Post D. Moiré Interferometry at VPI and SU. Exp Mech. 1983;23(2):203-10.

[27] Bhatt PM, Malhan RK, Rajendran P, Gupta SK. Building free-form thin shell parts using supportless extrusion-based additive manufacturing. Addit Manuf. 2020;32:101003.

[28] Fottenburg WG. Some Applications of Holographic Interferometry. Exp Mech. 1969;8:281-5.

[29] Wang YY, Chen DJ, Chiang FP. Material testing by computer aided speckle interferometry. Exp Tech. 1993;17(5):30-2.

[30] Bastawros AF, Voloshin AS. Thermal Strain Measurements in Electronic Packages through Fractional Fringe Moiré Interferometry. J Electron Packag. 1990;112(4):303-8.

[31] Bruck HA, McNeil SR, Sutton MA, Peters WH. Digital Image Correlation Using Newton-Raphson Method of Partial Differential Correction. Exp Mech. 1989;29(3):261-7.

[32] Zhang D, Zhang X, Cheng G. Compression strain measurement by digital speckle correlation. Exp Mech. 1999;39(1):62-5.

[33] Sutton MA, Wolters W], Peters WH, Ranson WF, McNeil SR. Determination of Displacements using an improved Digital Correlation method. Image Vis Comput. 1983;1(3):133-9.

[34] Sutton MA, Cheng MQ, Peters WH, Chao YJ, McNeill SR. Application of an Optimized Digital Correlation Method to Planar Deformation Analysis. Image Vis Comput. 1986;4(3):143-51.

[35] Sutton MA, Turner JL, Bruck HA, Chae TA. Full-field Representation of Discretely Sampled Surface Deformation for Displacement and Strain Analysis. Exp Mech. 1991;31(2):168-77.

[36] Sutton MA, McNeill SR, Jang J, Babai M. Effects of Subpixel Image Restoration on Digital Correlation Error. J Optical Eng. 1988;27(10):870-7.

[37] Spagnolo G, Schirripa Paoletti D, Ambrosini D, Guattari G. Electrooptic correlation for in situ diagnostics in mural frescoes. Pure \& Applied Optics. J Europ Optical Soc Part A. 1997;6(5):557-63.

[38] Manuello Bertetto A. Lightweight Structures Resisting by Form: The Grid Shells (in Italian); 2004. https://webthesis.bibl io.polito.it.

[39] Carpinteri A. Advanced Structural Mechanics. Taylor \& Francis Ltd; 2017. https://doi.org/10.1201/9781315375298.

[40] Misic T, Najdanovic-Lukic M, Nesic L. Dimensional analysis in physics and the Buckingham theorem. Eur J Phys. 2010;31(4):893-906.

[41] Piana G, Lofrano E, Manuello A, Ruta G, Carpinteri A. Compressive buckling for symmetric TWB with non-zero warping stiffness. Eng Struct. 2017;135:246-58.

[42] Piana G, Lofrano E, Manuello A, Ruta G. Natural frequencies and buckling of compressed non-symmetric thin-walled beams. Thinwalled Struct. 2017;111:189-96.

[43] Carpinteri A, Malvano R, Manuello A, Piana G. Fundamental frequency evolution in slender beams subjected to imposed axial displacements. J Sound Vibrat. 2014;333(11):2390-403.

[44] Manuello A. Semi-Rigid Connection in Timber Structure: Stiffness Reduction and Instability Interaction. Int J Struct Stab Dyn. 2020;20(7):2050072. 